\title{
Construction, Characterization, Estimation and Performance Analysis of Selection Generalized Nakagami Distributions with Environmental Applications
}

\author{
Mervat Mahdy ${ }^{\star}$, Dina Samir \\ Department of Statistics, Mathematics and Insurance, College of Commerce, Benha University, Egypt
}

\section{ARTICLE INFO}

\section{Article History}

Received 03 Aug 2018

Accepted 11 March 2019

\section{Keywords}

Nakagami distribution

Selection model

Distribution theory

Moment generating function

Log-likelihood function

Monte Carlo simulation

\begin{abstract}
In this article, a selection of Nakagami distribution is investigated. Some properties of the model with some plots of the density function are illustrated. Additionally, weighted of the one-sided Gaussian distribution, Generalized Rayleigh distribution are discussed as a special case of Generalized Nakagami distribution. In addition, maximum likelihood estimators are investigated with numerical methods and are compared by four sub-models with a real wave height data set. Finally, a simulation study is presented for parameters.
\end{abstract}

(C) 2020 The Authors. Published by Atlantis Press SARL. This is an open access article distributed under the CC BY-NC 4.0 license (http://creativecommons.org/licenses/by-nc/4.0/).

\section{INTRODUCTION}

Nakagami distribution $(N A)$ is useful for modeling the fading of radio signals and other areas of telecoms engineering. It has the applications in medical imaging investigation to model the ultrasounds especially in Echo (heart efficiency test). It is also helpful for modeling highfrequency seismogram envelopes. The reliability theory also make wide use of the NA distribution. By reason of the memory less property of this distribution, it is well appropriate to model the constant hazard rate portion; as it is used in reliability theory. Also, it is also used in the study of failure times of electrical components. On the other hand, the NA distribution is the excellence distribution to check the reliability of electrical component as compared to Gamma, Weibull and lognormal distributions.

The random variable $X$ has the NA distribution if its probability density function is defined as follows:

$$
g(x \mid \mu, \Omega)=2(\mu / \Omega)^{\mu} x^{2 \mu-1} \exp \left(-\mu x^{2} / \Omega\right) / \Gamma(\mu), \mu>0 ; \Omega>0, x>0,
$$

where $\mu$ is a shape parameter and $\Omega$ is a second parameter controlling spread (scale parameter). The cumulative distribution of a random variable from (1) can be obtained as

$$
G(x \mid \mu, \Omega)=\frac{1}{\Gamma(\mu)} \gamma\left(\mu, \mu x^{2} / \Omega\right)
$$

where $\gamma(.,$.$) is the incomplete gamma function. In Telatar [1], G(x \mid \mu, \Omega)$ is represented in terms of an incomplete gamma function dependent on the average signal-to-noise ratio $\left(\mu x^{2} / \Omega\right)$ which we denoted by ASNR. This allows the system to be characterized. The NA distribution covers a wide range of fading conditions. A special case of the NA distribution in which $\mu=0.5$ implies the one-sided Gaussian distribution $(O G)$. Also, when $\mu=1$, it implies the Rayleigh distribution $(R A)$. In addition, if $Y$ belongs to gamma distribution with shape and scale parameters $\theta_{1}$ and $\theta_{2}$ respectively, then $\sqrt{Y}$ belongs to NA distribution with parameters $\mu=\theta_{1}$ and $\Omega=\theta_{1} \theta_{2}$. Finally, if $2 \mu$ is integer-valued and if $B$ follows a chi distribution with parameters $2 \mu$, hence $\sqrt{(\Omega / 2 \mu) B} \in g(x \mid \mu, \Omega)$.

The problem of modeling lifetime of systems and components in reliability theory and survival analysis among other sciences is very important. In some real situations the measurements are not reported according to the standard distribution of the data. This may be due to the

*Corresponding author. Email: drmervat.mahdy@fcom.bu.edu.eg 
fact that the units of a population have unequal chances in order to be recorded by investigator. In a special case, the probability of recording data from the main population depends on size (length) of the data. The random sample drawn in this way is called a size-biased sample.

In this article, we pay attention to a selection model from the NA distribution. Selection distributions have been considered extensively by many authors; for a best survey of estimation method discussions and applications, see Heckman [2], Copas and Li [3], Arrellano-Valle et al. [4], Signer et al. [5], Balakrishnan et al. [6], Zamani et al. [7] and Arashi et al. [8] and more recently. The organization of this paper is as follows: In Section 2, we present definition and some representations of this model. In Section 3, some important properties are discussed. Also, numerical methods and graphs helped to picture some measures are obtained. Statistical inferences by numerical methods are fulfilled in Section 4 to estimate the parameters of the introduced distribution with real wave height data. In Section 5, the explanation of various estimators compared by performing the Monte Carlo simulation is studied.

We use the notation $X \stackrel{D}{\rightarrow} Y$ to mean that $X$ and $Y$ are equal in distribution.

\section{DEFINITION}

Let $\mathbf{A} \in \mathbf{R}^{+}$and $\mathbf{B} \in \mathbf{R}^{+}$be two random vectors and $\mathbf{D}$ to be a measurable subset of $\mathbf{R}^{+}$. Arellano-Valle et al. [4] studied a weighted distribution as the conditional distribution of $\mathbf{B}$ given $\mathbf{A} \in \mathbf{D}$. Clearly, a $p$-dimensional random vector $\mathbf{X}^{w}$ is said to have a multivariate weighted density function with parameters depending on the characteristics of $\mathbf{A}, \mathbf{B}$ and $\mathbf{D}$, if $\mathbf{X}^{w} \stackrel{D}{\rightarrow}(\mathbf{B} \mid \mathbf{A} \in \mathbf{D})$. If $\mathbf{B}$ has a pdf, $f_{\mathbf{B}}$, then $\mathbf{X}^{w}$ has a pdf as follows:

$$
f_{\mathbf{X}^{w}}(\mathbf{x})=f_{\mathbf{B}}(\mathbf{x}) \frac{\operatorname{Pr}(\mathbf{A} \in \mathbf{D} \mid \mathbf{B}=\mathbf{x})}{\operatorname{Pr}(\mathbf{A} \in \mathbf{D})} .
$$

The random variable $X^{w}$ is called the weighted version of $X$, and its distribution in relative to $X$ is called the weighted distribution of $X$ with weight function $w(x)$. Some special weighted functions are mentioned in Patil [9] and Azzalini [10].

Let $X$ be a random variable with the probability density function (1) and let we selected generalized weighted function as mentioned in Patil [9] as

$$
w(x)=x^{\theta} F^{i}(x) \bar{F}^{j}(x)
$$

Then, by (3) we have

$$
f_{\mathbf{X}^{w}}(\mathbf{x})=\frac{x^{\ominus} F^{i}(x) \bar{F}^{j}(x) g(x)}{E_{g}\left[x^{\ominus} F^{i}(x) \bar{F}^{j}(x)\right]} ; x>0 .
$$

According to (1), we have

$$
\begin{aligned}
I_{i, j, \theta}(\mu, \Omega)= & E_{g}\left[x^{\theta} F^{i}(x) \bar{F}^{j}(x)\right] \\
= & \frac{2(\mu / \Omega)^{\mu}}{\Gamma(\mu)} \int_{0}^{\infty}\left(\frac{1}{\Gamma(\mu)} \gamma\left(\mu, \mu x^{2} / \Omega\right)\right)^{i} \\
& \times\left(1-\frac{\gamma\left(\mu, \mu x^{2} / \Omega\right)}{\Gamma(\mu)}\right)^{j} x^{2 \mu+\theta-1} \exp \left(-\mu x^{2} / \Omega\right) d x
\end{aligned}
$$

As mentioned in Gradshteyn and Ryshik [11], we know that the lower incomplete gamma function can be rewritten as

$$
\gamma(\alpha, x)=\frac{x^{\alpha}}{\alpha} \times_{1} F_{1}(\alpha ; 1+\alpha ;-x)
$$

According to Gradshteyn and Ryshik [11] $\alpha$ and $x$ are parameters and ${ }_{1} F_{1}(. ; . ;$.) is the generalized hypergeometric function with one parameter of type 1 and one parameter of type 2. Suppose for a reasonably low outage probability, the ASNR has to be sufficiently large, that is, the term $\mu x^{2} / \Omega$ in (2) has to be small, we invoke an asymptotic expansion of the confluent hypergeometric function at small $x$. From the series expansion in Gradshteyn and Ryshik [11], we observe that ${ }_{1} F_{1}(\alpha ; 1+\alpha ;-x) \rightarrow 1$ for $x \rightarrow 0$. By taking $\gamma\left(\mu, \mu x^{2} / \Omega\right) / \Gamma(\mu)=u$, we get that (5) can be expressed as

$$
I_{i, j, \theta}(\mu, \Omega)=\left(\frac{\Omega}{\mu}\right)^{\frac{\theta}{2}}(\Gamma(\mu+1))^{\frac{\theta}{2 \mu}} B\left(j+1, i+\frac{\theta}{2 \mu}+1\right),
$$


whereas $B(.,$.$) is beta function. By using (4) and (6), and after some elementary algebra, we have Generalized Nakagami density function,$ $\mathrm{GN}$, (denoted as $G N(i, j, \theta, \mu, \Omega))$ as follows:

$$
f_{\mathbf{X}^{w}}(\mathbf{x})=\varphi_{1}(\mu, \theta, \Omega) \varphi_{2}^{-1}(\mu, \theta, \Omega), x>0
$$

where

$\varphi_{1}(\mu, \theta, \Omega)=2\left(\gamma\left(\mu, \mu x^{2} / \Omega\right)\right)\left(\Gamma(\mu)-\gamma\left(\mu, \mu x^{2} / \Omega\right)\right)^{j} x^{2 \mu+\theta-1} \exp \left(-\mu x^{2} / \Omega\right)$

$\varphi_{2}(\mu, \theta, \Omega)=(\Omega)^{\frac{\theta}{2}+\mu}(\mu) \frac{\theta(1-\mu)-2 \mu^{2}}{2 \mu} \Gamma^{i+j+\frac{\theta}{2 \mu}+1}(\mu) B\left(j+1, i+\frac{\theta}{2 \mu}+1\right)$.

In addition, we can conclude that a generalized OG distribution (denoted as $\left.f_{\mathbf{X}^{w}}^{[1]}(\mathbf{x})\right)$ and a Generalized RA distribution $\left(\operatorname{denoted}\right.$ as $\left.f_{\mathbf{X}^{w}}^{[2]}(\mathbf{x})\right)$ as sub-models of (7) as respectively follows:

$$
f_{\mathbf{X}^{w}}^{[1]}(\mathbf{x})=\pi^{-\frac{j+\theta+1}{2}} \varphi_{3}(\mu, \theta, \Omega) \varphi_{4}^{-1}(\mu, \theta, \Omega), x \in R^{+}
$$

and

$$
f_{\mathbf{X}^{w}}^{[2]}(\mathbf{x})=\varphi_{4}^{-1}(\mu, \theta, \Omega) \varphi_{5}(\mu, \theta, \Omega), x \in R^{+},
$$

where $\operatorname{erf}(x)$ is error function,

$\varphi_{3}(\mu, \theta, \Omega)=(2)^{\frac{\theta+1}{2}}(\operatorname{erf}(x \sqrt{1 / 2 \Omega}))^{i}(\sqrt{\pi}-\sqrt{\pi} \operatorname{erf}(x \sqrt{1 / 2 \Omega}))^{j} x^{\theta} \exp \left(-x^{2} / 2 \Omega\right)$,

$\varphi_{4}(\mu, \theta, \Omega)=\Omega^{\frac{\theta+1}{2}} B(j+1, i+\theta+1)$ and

$\varphi_{5}(\mu, \theta, \Omega)=2\left(\gamma\left(1, x^{2} / \Omega\right)\right)^{i}\left(1-\gamma\left(1, x^{2} / \Omega\right)\right)^{j} x^{\theta+1} \exp \left(-x^{2} / \Omega\right)$.

Now, we consider the effect of any parameter on the pdf of $G N(i, j, \theta, \mu, \Omega)$ introduced in (7) with Figure 1 . In each panel, one of the parameters changes in first three panels and in the last panel we change all parameters with together.
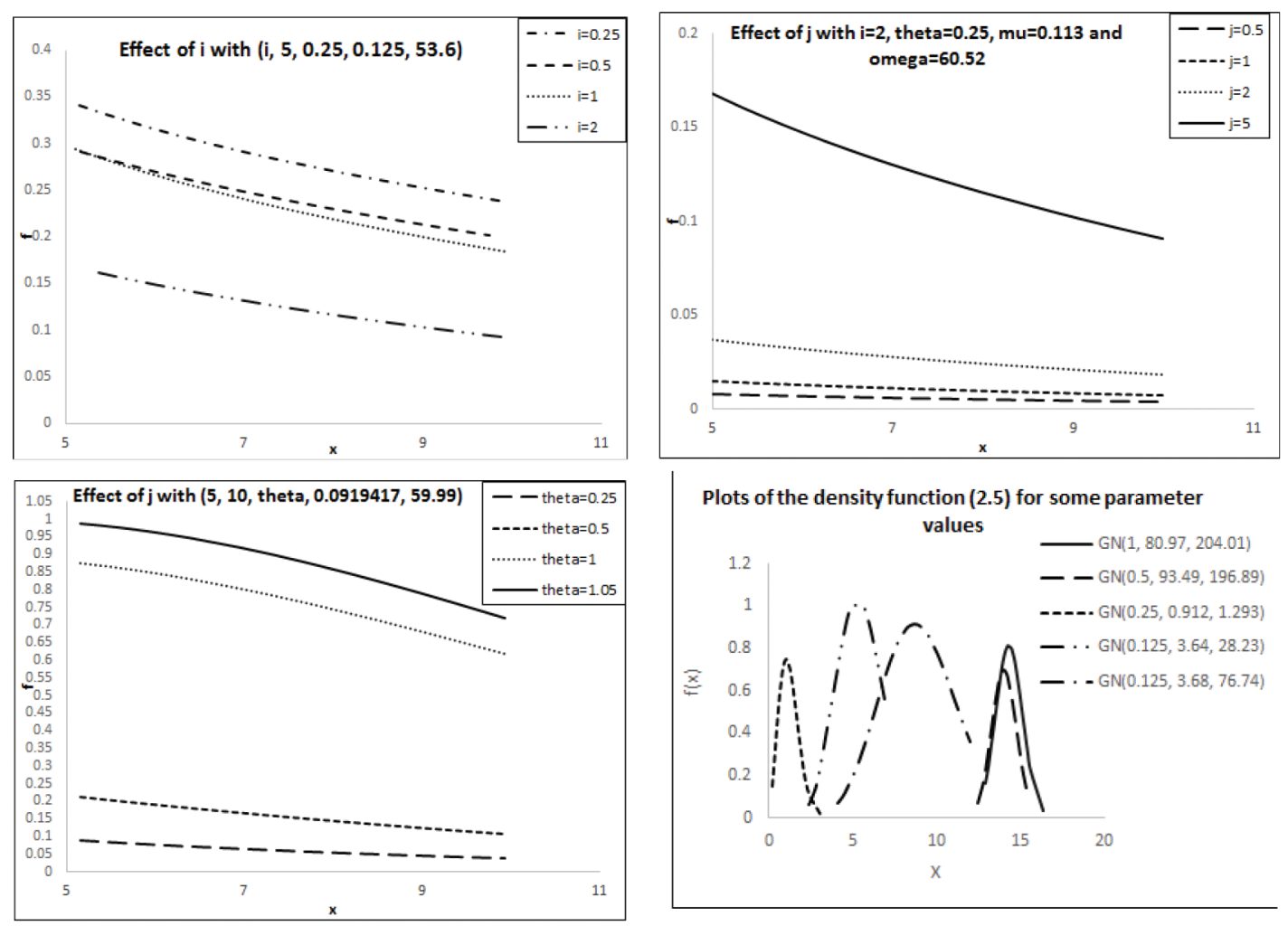

Figure $1 \mid$ Plots of the density function with effect of parameters. 
We furnish two simple formula for pdf of the $G N(i, j, \theta, \mu, \Omega)$ distribution, if $j \notin \mathbb{Z}_{>0}$ or $j \in \mathbb{Z}_{>0}$. First, if $|x|<1$ and $j \notin \mathbb{Z}_{+}$, it follows the series (Nadarajah and Kotz [12], p. 324, Eq. (1.7))

$$
(1-x)^{j}=\sum_{y=0}^{\infty} \frac{(-1)^{y} \Gamma(j+1)}{\Gamma(j-y+1) y !} x^{y}
$$

Using the series representation (8), the pdf of the $G N(i, j, \theta, \mu, \Omega)$ distribution for $\left|\gamma\left(\mu, \mu x^{2} / \Omega\right) / \Gamma(\mu)\right|<1$ and $j \notin \mathbb{Z}_{+}$can be expanded as

$$
f_{\mathbf{X}^{w}}(\mathbf{x})=2 \varkappa_{2}^{-1}(x, \theta, \mu, \Omega) \sum_{y=0}^{\infty} \frac{(-1)^{y} \Gamma(j+1)}{\Gamma^{y}(\mu) \Gamma(j-y+1) y !} \frac{\left(\mu x^{2} / \Omega\right)^{\mu i+y}}{\mu^{i+y}} \times \varkappa_{1}(x, \theta, \mu, \Omega),
$$

where

$\chi_{1}(x, \theta, \mu, \Omega)=\left({ }_{1} F_{1}\left(\mu ; 1+\mu ;-\left(\mu x^{2} / \Omega\right)\right)\right)^{i+y} x^{2 \mu+\theta-1} \exp \left(-\mu x^{2} / \Omega\right) ;$

$\chi_{2}(x, \theta, \mu, \Omega)=(\Omega)^{\frac{\theta}{2}+\mu}(\mu) \frac{\theta(1-\mu)-2 \mu^{2}}{2 \mu} \Gamma^{i+\frac{\theta}{2 \mu}+1}(\mu) B\left(j+1, i+\frac{\theta}{2 \mu}+1\right)$.

By using confluent hypergeometric function Rainville ([13], p. 123) we can be rewrite the pdf of the $G N(i, j, \theta, \mu, \Omega)$ distribution as

$$
f_{\mathbf{X}^{w}}(\mathbf{x})=\frac{2 \sum_{y=0}^{\infty} \frac{(-1)^{y} \Gamma(j+1)}{\Gamma y(\mu) \Gamma(j-y+1) y !} \frac{\left(\mu x^{2} / \Omega\right)^{\mu i+y}}{\mu^{i+y}} \times\left(\sum_{n=0}^{\infty} \frac{(\mu)_{n}}{(\mu+1)_{n}} \frac{\left(-\left(\mu x^{2} / \Omega\right)\right)^{n}}{n !}\right)^{i+y} x^{2 \mu+\theta-1} \exp \left(-\mu x^{2} / \Omega\right)}{(\Omega)^{\frac{\theta}{2}+\mu}(\mu) \frac{\theta(1-\mu)-2 \mu^{2}}{2 \mu} \Gamma^{i+\frac{\theta}{2 \mu}+1}(\mu) B\left(j+1, i+\frac{\theta}{2 \mu}+1\right)} .
$$

If $\left|\left((\mu)_{n}\left(-\left(\mu x^{2} / \Omega\right)\right)^{n}\right) /\left((\mu+1)_{n} n !\right)\right|<1$ and $(n)_{a}$ is Pochhammer's symbol, Eq. (9) can be rewritten as

$$
f_{\mathbf{X}^{w}}(\mathbf{x})=\frac{2 \sum_{y=0}^{\infty} \sum_{n=0}^{\infty} \varphi_{\mu, j}(y, n) x^{2 \mu+\theta+2 n^{2}-1+2 \mu i+2 y} \exp \left(-\mu x^{2} / \Omega\right)}{(\mu / \Omega)^{-\mu} \Gamma^{i+1}(\mu) I_{i, j, \theta}(\mu, \Omega)},
$$

where

$$
\varphi_{\mu, j}(y, n)=\frac{(-1)^{y+n^{2}} \Gamma(j+1)(n+1)_{(i+y-1)}}{\Gamma^{y}(\mu) \Gamma(j-y+1) y !(i+y-1) !} \frac{(\mu / \Omega)^{\mu i+y+n^{2}}}{(n !)^{n} \mu^{i+y}}\left(\frac{(\mu)_{n}}{(\mu+1)_{n}}\right)^{n} .
$$

Now, we can obtain cumulative distribution function of the $G N(i, j, \theta, \mu, \Omega)$ in this case as follows:

$$
F_{\mathbf{X}^{w}}(\mathbf{x})=\frac{2 \sum_{y=0}^{\infty} \sum_{n=0}^{\infty} \varphi_{\mu, j}(y, n) \int_{0}^{x} u^{2 \mu+\theta+2 n^{2}-1+2 \mu i+2 y} \exp \left(-\mu u^{2} / \Omega\right) d u}{(\mu / \Omega)^{-\mu} \Gamma^{i+1}(\mu) I_{i, j, \theta}(\mu, \Omega)} .
$$

Now, on setting $t=\mu u^{2} / \Omega, F_{\mathbf{X}^{w}}(\mathbf{x})$ reduces to

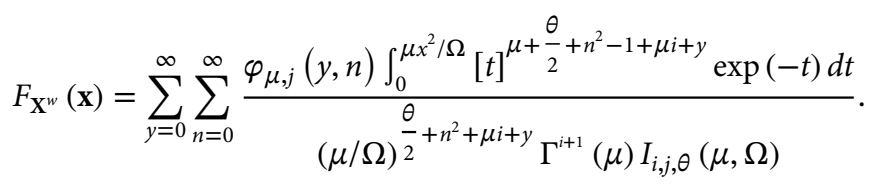

Then we have

$$
F_{\mathbf{X}^{w}}(\mathbf{x})=\sum_{y=0}^{\infty} \sum_{n=0}^{\infty} \frac{\varphi_{\mu, j}(y, n) \gamma\left(\mu(i+1)+\frac{\theta}{2}+n^{2}+y, \mu x^{2} / \Omega\right)}{(\mu / \Omega)^{\frac{\theta}{2}+n^{2}+\mu i+y} \Gamma^{i+1}(\mu) I_{i, j, \theta}(\mu, \Omega)} .
$$


If $\mu+\frac{\theta}{2}+n^{2} \in \mathbb{Z}_{+}$, then

$$
F_{\mathbf{X}^{w}}(\mathbf{x})=\sum_{y=0}^{\infty} \sum_{n=0}^{\infty} \frac{\varphi_{\mu, j}(y, n)\left(\mu+\frac{\theta}{2}+n^{2}-1\right) !\left(1-\exp \left(-\mu x^{2} / \Omega\right) \sum_{w=0}^{\mu(i+1)+\frac{\theta}{2}+n^{2}+y-1}\left(\mu x^{2} / \Omega\right)^{w} / w !\right)}{(\mu / \Omega)^{\frac{\theta}{2}+n^{2}+\mu i+y} \Gamma^{i+1}(\mu) I_{i, j, \theta}(\mu, \Omega)} .
$$

Second, by application of the binomial expansion in (7), when $j \in \mathbb{Z}_{+}$, we can rewrite (7) as follows:

$$
f_{\mathbf{X}^{w}}(\mathbf{x})=\frac{2 \sum_{k=0}^{j}(-1)^{k}\left(\begin{array}{l}
j \\
k
\end{array}\right)(1 / \Gamma(\mu))^{k+i+1}\left(\gamma\left(\mu, \mu x^{2} / \Omega\right)\right)^{k+i} x^{2 \mu+\theta-1} \exp \left(-\mu x^{2} / \Omega\right)}{(\mu / \Omega)^{-\mu} I_{i, j, \theta}(\mu, \Omega)} .
$$

With same assumptions in (10) and same steps, we can represent (12) as follow:

$$
f_{\mathbf{X}^{w}}(\mathbf{x})=\frac{2 \sum_{k=0}^{j} \sum_{n=0}^{\infty} \varpi_{\mu, j, i}(n, k)\left(\frac{(\mu)_{n}}{n !(\mu+1)_{n}}\right)^{n} x^{2 \mu(k+i)+2 \mu+\theta-1+2 n^{2}} \exp \left(-\mu x^{2} / \Omega\right)}{I_{i, j, \theta}(\mu, \Omega)},
$$

where $\varpi_{\mu, j, i}(n, k)$ is given in Appendix.

Also, the cumulative distribution function in this case can be followed with same technique.

$$
F_{\mathbf{X}^{w}}(\mathbf{x})=\frac{\sum_{k=0}^{j} \sum_{n=0}^{\infty} \varpi_{\mu, j, i}(n, k)\left(\frac{(\mu)_{n}}{n !(\mu+1)_{n}}\right)^{n}(\Omega / \mu)^{\mu(k+i)+\mu+\frac{\theta}{2}+n^{2}} \gamma\left(\left(\mu(k+i)+\mu+\frac{\theta}{2}+n^{2}\right), \mu x^{2} / \Omega\right)}{I_{i, j, \theta}(\mu, \Omega)} .
$$

If $\mu(k+i)+\mu+\frac{\theta}{2}+n^{2} \in \mathbb{Z}_{+}$, we have

$$
F_{\mathbf{X}^{w}}(\mathbf{x})=\frac{\sum_{k=0}^{j} \sum_{n=0}^{\infty} \varpi_{\mu, j, i}(n, k) c_{\mu, \Omega, i}(n, k)\left(1-\exp \left(-\mu x^{2} / \Omega\right) \sum_{w=0}^{\left(\mu(k+i)+\mu+\frac{\theta}{2}+n^{2}-1\right)}\left(\mu x^{2} / \Omega\right)^{w} / w !\right)}{I_{i, j, \theta}(\mu, \Omega)},
$$

where $c_{\mu, j, i}(n, k)=\left(\frac{(\mu)_{n}}{n !(\mu+1)_{n}}\right)^{n}(\Omega / \mu)^{\mu(k+i)+\mu+\frac{\theta}{2}+n^{2}}\left(\mu(k+i)+\mu+\frac{\theta}{2}+n^{2}-1\right) !$.

\section{PROPERTIES OF THE GN DISTRIBUTION}

We need to emphasize the importance of hazard rate, reversed hazard rate, moments and moment generating functions in any statistical analysis especially in applied issues. An advantages and characteristics of a distribution can be studied through measures of central tendency; dispersion functions; measures of skewness and kurtosis.

The hazard rate $\left(r(x)=f_{\mathbf{X}^{w}}(\mathbf{x}) /\left(1-F_{\mathbf{X}^{w}}(\mathbf{x})\right)\right)$, and reversed hazard rate functions $\left(\tilde{r}(x)=f_{\mathbf{X}^{w}}(\mathbf{x}) / F_{\mathbf{X}^{w}}(\mathbf{x})\right)$ of $G N(i, j, \theta, \mu, \Omega)$ can be obtained directly using Equations $(10-12,14)$.

Now, we consider the effect of any parameter on reversed hazard function with Figure 2. In each panel, one of the parameters is changed. If $X \sim G N(i, j, \theta, \mu, \Omega)$, then the $r$ th moment is given by

$$
E\left(X^{r}\right)=\frac{\sum_{y=0}^{\infty} \sum_{n=0}^{\infty} \varphi_{\mu, j}(y, n) \Gamma\left(\mu(i+1)+\frac{\theta+r}{2}+n^{2}+y\right)}{\Gamma^{i+1}(\mu) I_{i, j, \theta}(\mu, \Omega)} .
$$

If $\left|\left((\mu)_{n}\left(-\left(\mu x^{2} / \Omega\right)\right)^{n}\right) /\left((\mu+1)_{n} n !\right)\right|<1,\left|\gamma\left(\mu, \mu x^{2} / \Omega\right) / \Gamma(\mu)\right|<1, j \notin \mathbb{Z}_{+}$and

$$
\tau_{\mu, j}(y, n)=\frac{(-1)^{y+n^{2}} \Gamma(j+1)(n+1)_{(i+y-1)}}{\Gamma^{y}(\mu) \Gamma(j-y+1) y !(i+y-1) !} \frac{[(\Omega / \mu)]^{\frac{\theta}{2}+\frac{r}{2}}}{(n !)^{n} \mu^{i+y}}\left(\frac{(\mu)_{n}}{(\mu+1)_{n}}\right)^{n} .
$$



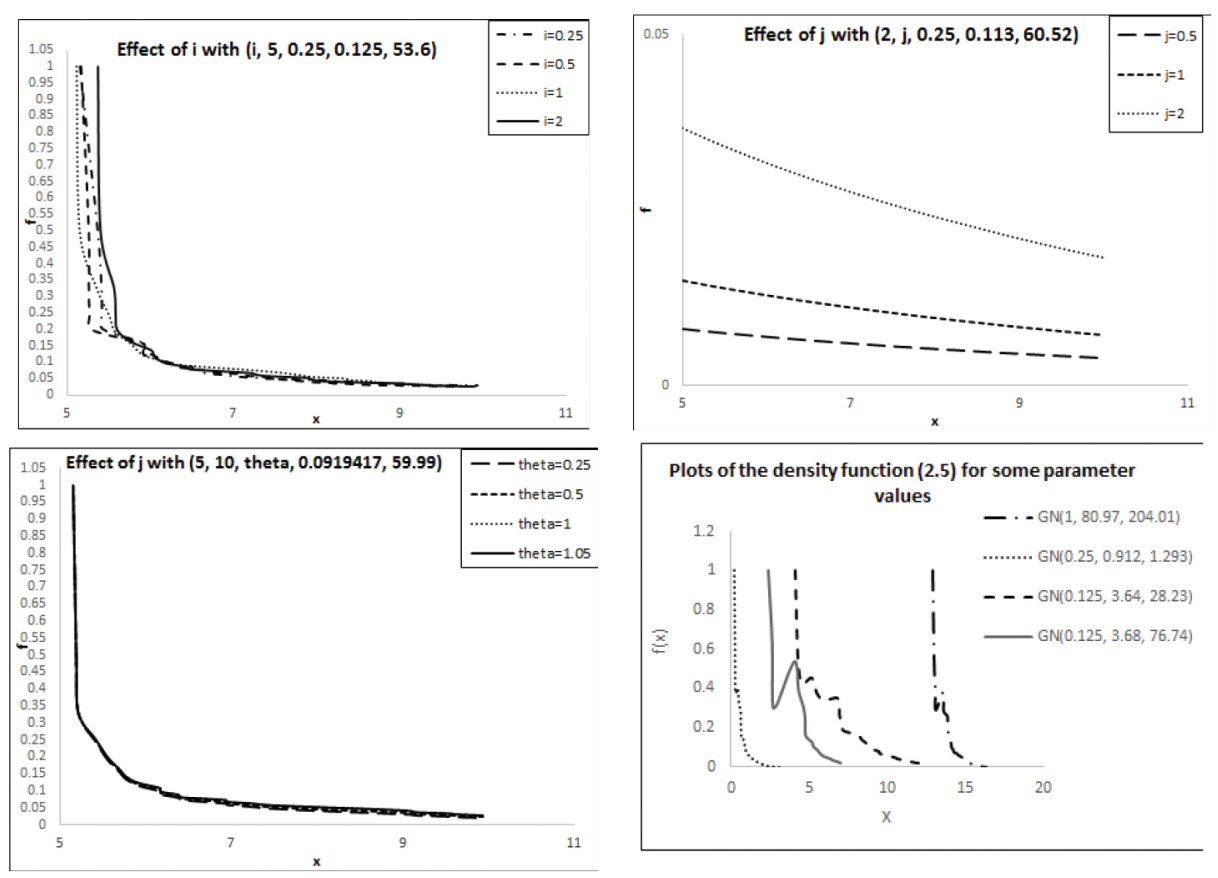

Figure 2 Plots of reversed hazard function with effect of parameters.

Also, if $j \in \mathbb{Z}_{+}$, we obtain $E\left(X^{r}\right)$ as

$$
E\left(X^{r}\right)=\sum_{k=0}^{j} \sum_{n=0}^{\infty} \bar{\varpi}_{\mu, j, i}(n, k) \Gamma\left(\mu(k+i)+\mu+\frac{\theta}{2}+n^{2}\right)
$$

where $\bar{\varpi}_{\mu, j, i}(n, k)=\varpi_{\mu, j, i}(n, k)\left((\mu)_{n} /\left(n !(\mu+1)_{n}\right)\right)^{n} /\left([(\mu / \Omega)]^{\mu(k+i)+\mu+\frac{\theta}{2}+n^{2}} I_{i, j, \theta}(\mu, \Omega)\right)$. Now, The skewness and kurtosis measures can be obtained from the ordinary moments utilizing well-known relationships.

If $X \sim G N(i, j, \theta, \mu, \Omega)$, then its moment generating function is given by

$$
M(t)=\frac{2 \sum_{y=0}^{\infty} \sum_{n=0}^{\infty} \varphi_{\mu, j}(y, n) \int_{0}^{\infty} x^{2 \mu+\theta+2 n^{2}-1+2 \mu i+2 y} \exp \left(-\mu x^{2} / \Omega+t x\right) d x}{(\mu / \Omega)^{-\mu} \Gamma^{i+1}(\mu) I_{i, j, \theta}(\mu, \Omega)} .
$$

Substituting $u=\mu x^{2} / \Omega+t x$, we have

$$
M(t)=\sum_{y=0}^{\infty} \sum_{n=0}^{\infty} \frac{2 \varphi_{\mu, j}(y, n) \int_{0}^{\infty}\left(t^{2}+4(\mu / \Omega) u\right)^{-0.5}\left[t \pm \sqrt{t^{2}+4(\mu / \Omega) u}\right]^{2 \mu+\theta+2 n^{2}-1+2 \mu i+2 y} \exp (-u) d u}{[2(\mu / \Omega)]^{2 \mu+\theta+2 n^{2}-1+2 \mu i+2 y}(\mu / \Omega)^{-\mu} \Gamma^{i+1}(\mu) I_{i, j, \theta}(\mu, \Omega)} .
$$

Hence, for $2 \mu+\theta+2 n^{2}-1+2 \mu i+2 y>0$ integer, and by taking $2 \mu+\theta+2 n^{2}-1+2 \mu i+2 y=d$, and $d \in \mathbb{Z}_{+}$we can obtain

$$
\begin{aligned}
M(t) & =\sum_{y=0}^{\infty} \sum_{n=0}^{\infty} \frac{2 \varphi_{\mu, j}(y, n) \sum_{s=0}^{d}( \pm 1)^{s}\left(\begin{array}{c}
d \\
s
\end{array}\right) t^{d-s} \int_{0}^{\infty}\left(4 u(\mu / \Omega)+t^{2}\right)^{\frac{s-1}{2}} \exp (-u) d u}{[2(\mu / \Omega)]^{2 \mu+\theta+2 n^{2}-1+2 \mu i+2 y}(\mu / \Omega)^{-\mu} \Gamma^{i+1}(\mu) I_{i, j, \theta}(\mu, \Omega)}, \\
& =\sum_{y=0}^{\infty} \sum_{n=0}^{\infty} \sum_{s=0}^{d} \frac{\left(\begin{array}{c}
d \\
s
\end{array}\right) 2 \varphi_{\mu, j}(y, n)( \pm 1)^{s} t^{d-s}(4(\mu / \Omega))^{\frac{s-1}{2}} \Gamma\left(\frac{s+1}{2}, \frac{t^{2}}{4(\mu / \Omega)}\right) \exp \left(\left[\frac{t^{2}}{4(\mu / \Omega)}\right]\right)}{[2(\mu / \Omega)]^{2 \mu+\theta+2 n^{2}-1+2 \mu i+2 y}(\mu / \Omega)^{-\mu} \Gamma^{i+1}(\mu) I_{i, j, \theta}(\mu, \Omega)} .
\end{aligned}
$$

It is not possible to compute the median and mode explicitly. The mode is obtained from the equation below

$$
\left(\mu x^{2} / \Omega\right)^{\mu-1} e^{-\mu x^{2} / \Omega}\left[i\left(\gamma\left(\mu, \mu x^{2} / \Omega\right)\right)^{-1}-j\left(\Gamma(\mu)-\gamma\left(\mu, \mu x^{2} / \Omega\right)\right)^{-1}\right]=2(\mu x / \Omega)-(2 \mu+\theta-1) x^{-1} .
$$


To obtain the median, we consider that $F(x)=\frac{1}{2}$, hence

$$
\sum_{y=0}^{\infty} \sum_{n=0}^{\infty} \frac{\varphi_{\mu, j}(y, n)\left(\mu+\frac{\theta}{2}+n^{2}-1\right) !\left(1-\exp \left(-\mu x^{2} / \Omega\right) \sum_{w=0}^{\mu(i+1)+\frac{\theta}{2}+n^{2}+y-1}\left(\mu x^{2} / \Omega\right)^{w} / w !\right)}{(\mu / \Omega)^{\frac{\theta}{2}+n^{2}+\mu i+y} \Gamma^{i+1}(\mu) I_{i, j, \theta}(\mu, \Omega)}=1 / 2
$$

if $\mu+\frac{\theta}{2}+n^{2} \in \mathbb{Z}_{+}$, otherwise we should solve the following equation to obtain the median:

$$
\sum_{k=0}^{j} \sum_{n=0}^{\infty} \varpi_{\mu, j, i}(n, k) c_{\mu, \Omega,, i}(n, k)\left(1-\exp \left(-\mu x^{2} / \Omega\right) \sum_{w=0}^{d}\left(\mu x^{2} / \Omega\right)^{w} / w !\right)=I_{i, j, \theta}(\mu, \Omega) / 2,
$$

where $d=\left(\mu(k+i)+\mu+\frac{\theta}{2}+n^{2}-1\right)$.

These central indices computed numerically, and we show output for some values that indicate in the Table 1 as

The basic uncertainty measure for density function $f$ is differential entropy

$$
H_{X}(f)=E\left[-\ln f_{X}(X)\right]=\int_{0}^{\infty} f_{X}(x) \ln \frac{1}{f_{X}(x)} d x
$$

The differential entropy of a non-negative absolutely continuous random variable $X$, is also known as Shannon information measure or sometime called dynamic measure of uncertainty. Intuitively speaking the entropy gives the expected uncertainty contained in $f(x)$ about the predictability of an outcome of $X$, see Ebrahimi and Pellerey [14]. It also measure how the distribution spreads over its domain. A high value of $H_{X}$ corresponds to a low concentration of the probability mass of $X$.

When $\theta=1, i=j=0$, the density function in (7) is referred to as length-biased distribution. Then, we have

$$
E\left[x^{\theta}\right]=\frac{1}{\Gamma(\mu)}\left(\frac{\Omega}{\mu}\right)^{\frac{\theta}{2}} \Gamma\left(\mu+\frac{\theta}{2}\right) .
$$

Hence,

$$
H_{X}\left(f_{\mathbf{X}^{w}}(\mathbf{x})\right)=\ln 2+\left(\mu+\frac{\theta}{2}\right)[\ln (\mu / \Omega)-1]+\frac{(2 \mu+\theta-1)}{2}\left[\psi\left(\mu+\frac{\theta}{2}\right)-\ln \left(\frac{\mu}{\Omega}\right)\right]-\ln \Gamma\left(\mu+\frac{\theta}{2}\right),
$$

where

$$
\int_{0}^{\infty} t^{v-1} \ln t \exp (-p t) d t=\Gamma(v) p^{-v}[\psi(v)-\ln p]
$$

Also, Havrda and Charvat [15] introduced $\beta$-entropy class as follows:

$$
H_{\beta}\left(f_{\mathbf{X}^{w}}(\mathbf{x})\right)=\left\{\begin{array}{cl}
\frac{1}{\beta-1}\left[1-\int_{0}^{\infty} f_{\mathbf{X}^{w}}^{\beta}(\mathbf{x})(x) d x\right] ; & \beta \neq 1, \beta>0, \\
H\left(f_{\mathbf{X}^{w}}(\mathbf{x})\right) & \beta=1,
\end{array}\right.
$$

Table 1 The mean, median and mode of $G N(\theta, \mu, \Omega)$.

\begin{tabular}{lrrr}
\hline $\boldsymbol{\theta}, \boldsymbol{\mu}, \boldsymbol{\Omega})$ & Mean & Median & Mode \\
\hline$(1,80.97,204.01)$ & 14.2838 & 14.1990 & 14.2357 \\
$(0.5,93.49,196.89)$ & 14.0072 & 13.9790 & 14.0198 \\
$(0.25,0.912,1.293)$ & 1.0091 & 0.9493 & 1.0548 \\
$(0.125,3.68,76.74)$ & 5.4827 & 5.4178 & 4.9304 \\
$(0.125,3.64,28.23)$ & 8.9588 & 8.8974 & 8.6173 \\
\hline
\end{tabular}


where $\beta$ is a non-stochastic constant, by using (15) and (7) and after some elementary algebra, we have:

$$
\begin{aligned}
& \int_{0}^{\infty} x^{\theta \beta} G^{i \beta}(x) \bar{G}^{j \beta}(x) g^{\beta}(x \mid \mu, \Omega) d x \\
= & \left(\frac{\Omega}{\mu}\right)^{\frac{\beta \theta}{2}}(\Gamma(\mu+1))^{\frac{\beta \theta}{2 \mu}} B\left(j \beta+1, \beta\left(i+\frac{\theta}{2 \mu}\right)\right) .
\end{aligned}
$$

Hence

$$
H_{\beta}\left(f_{\mathbf{X}^{w}}(\mathbf{x})\right)=\frac{1}{\beta-1}\left[1-\frac{B\left(j \beta+1, \beta\left(i+\frac{\theta}{2 \mu}\right)\right)}{B^{\beta}\left(j+1, i+\frac{\theta}{2 \mu}+1\right)}\right]
$$

for all $\beta>1$.

\section{NUMERICAL ILLUSTRATION}

In this section, we consider the problem of statistical inference about generalized weighted of the NA distribution such as maximum likelihood estimator (MLE) of the unknown parameters, asymptotic distribution and bootstrap confidence intervals and bias reduced maximum likelihood.

\subsection{MLE and Asymptotic Distribution}

Let $X_{1}, X_{2}, \ldots, X_{N}$ be random variables that are i.i.d. according to $(7)$ and $\Theta=(i, j, \theta, \mu, \Omega)^{T}$. The log-likelihood function of the independent multivariate generalized weighted of the NA distribution based on $X_{1}, X_{2}, \ldots, X_{N}$ is given by

$$
\begin{aligned}
l_{n}= & \sum_{i=1}^{N} \ln g^{w_{\theta, i, j}}(x) \\
& \quad i \sum_{k=1}^{N} \ln \gamma\left(\mu, \mu x_{k}^{2} / \Omega\right)+j \sum_{k=1}^{N} \ln \left(\Gamma(\mu)-\sum_{k=1}^{N} \gamma\left(\mu, \mu x_{k}^{2} / \Omega\right)\right) \\
= & \quad+(2 \mu+\theta-1) \sum_{k=1}^{N} \ln x_{k}-\sum_{k=1}^{N} \mu x_{k}^{2} / \Omega+N \ln 2-N\left(\frac{\theta}{2}+\mu\right) \ln \Omega \\
& -N\left(\frac{\theta(1-\mu)-2 \mu^{2}}{2 \mu}\right) \ln \mu-N\left(i+j+\frac{\theta}{2 \mu}+1\right) \ln \Gamma(\mu)-N \ln B\left(j+1, i+\frac{\theta}{2 \mu}+1\right)
\end{aligned}
$$

where $\left\{x_{k}, k=1, \ldots, N\right\}$ are samples of $\left\{X_{k}, k=1, \ldots, N\right\}$, and we simply apply the chain rule:

$$
\frac{\partial}{\partial x} \ln \Gamma(x+k)=-\gamma+\sum_{r=1}^{\infty}\left(\frac{1}{r}-\frac{1}{r+x+k-1}\right)
$$

where $\gamma$ is the Euler-Mascheroni constant. Let $C_{u}=(\mu)^{\mu-1}\left(x_{u}^{2} / \Omega\right)^{\mu} \exp \left(-\mu x_{u}^{2} / \Omega\right), \zeta=\Gamma(\mu)-\sum_{k=1}^{N} \gamma\left(\mu, \mu x_{k}^{2} / \Omega\right)$ and $\psi_{x}^{\text {tr }}()=$. $\partial \psi_{0}(.) / \partial x$ denotes the trigamma function, then the score function is given by $U\left(X_{1}, X_{2}, \ldots, X_{N} \mid \Theta=\left(\frac{\partial l_{n}}{\partial i}, \frac{\partial l_{n}}{\partial j}, \frac{\partial l_{n}}{\partial \theta} \text {, } \frac{\partial l_{n}}{\partial \mu}, \frac{\partial l_{n}}{\partial \Omega}\right)^{T}\right.$, where

$$
\begin{gathered}
\frac{\partial l_{n}}{\partial i}=\sum_{k=1}^{N} \ln \gamma\left(\mu, \mu x_{k}^{2} / \Omega\right)-N \ln \Gamma(\mu)-N\left[\psi_{0}\left(i+\frac{\theta}{2 \mu}+1\right)-\psi_{0}\left(i+\frac{\theta}{2 \mu}+j+2\right)\right], \\
\frac{\partial l_{n}}{\partial j}=\sum_{k=1}^{N} \ln \zeta-N \ln \Gamma(\mu)-N\left[\psi_{0}(j+1)-\psi_{0}\left(i+j+\frac{\theta}{\mu}+2\right)\right],
\end{gathered}
$$




$$
\begin{gathered}
i \sum_{k=1}^{N} \frac{C_{k}}{\gamma\left(\mu, \mu x_{k}^{2} / \Omega\right)}+\frac{N j\left[\psi(\mu) \Gamma(\mu)-\sum_{k=1}^{N} C_{k}\right]}{\zeta}+2 \sum_{k=1}^{N} \ln x_{k}-\sum_{k=1}^{N} x_{k}^{2} / \Omega-N \ln \Omega \\
\frac{\partial l_{n}}{\partial \mu}=-N\left[\frac{\ln \mu\left(-4 \mu^{2}-2 \theta\right)}{4 \mu^{2}}+\frac{\theta(1-\mu)-2 \mu^{2}}{2 \mu^{2}}\right]-\ln \Gamma(\mu) \frac{-\theta N}{2 \mu^{2}}-N \psi_{0}(\mu)\left(i+j+\frac{\theta}{2 \mu}+1\right) \\
+N \frac{\theta}{2 \mu^{2}}\left[\psi_{0}\left(i+\frac{\theta}{2 \mu}+1\right)-\psi_{0}\left(i+j+\frac{\theta}{2 \mu}+2\right)\right] \\
\frac{\partial l_{n}}{\partial \Omega}=\sum_{k=1}^{N} \mu x_{k}^{2} / \Omega^{2}-i \Omega^{2 \mu-2} \mu^{-\mu+2} \sum_{k=1}^{N} \frac{\left(x_{k}^{2}\right)^{1-\mu} C_{k}}{\gamma\left(\mu, \mu x_{k}^{2} / \Omega\right)}+j N \Omega^{2 \mu-2} \mu^{-\mu+2} \frac{\sum_{k=1}^{N} C_{k} x_{k}^{2(1-\mu)}}{\zeta}-\frac{N}{\Omega}\left(\frac{\theta}{2}+\mu\right), \\
\frac{\partial l_{n}}{\partial \theta}=\sum_{k=1}^{N} \ln x_{k}-(N \ln \Omega / 2)-N \ln \mu(1-\mu) / 2 \mu-N \ln \Gamma(\mu) / 2 \mu \\
-N\left[\begin{array}{c}
\left(2 \mu\left(i+\frac{\theta}{2 \mu}\right)\right)^{-1}+2 \mu\left(-\gamma+\sum_{r=1}^{\infty}\left(\frac{1}{r}-\frac{1}{r+i+\frac{\theta}{2 \mu}-1}\right)\right)-\left[2 \mu\left(j+i+\frac{\theta}{2 \mu}+1\right)\right] \\
-\left(2 \mu\left(j+i+\frac{\theta}{2 \mu}\right)\right)^{-1}-2 \mu\left(-\gamma+\sum_{r=1}^{\infty}\left(\frac{1}{r}-\frac{1}{r+j+i+\frac{\theta}{2 \mu}-1}\right)\right)
\end{array}\right],
\end{gathered}
$$

and $\psi_{n}(x)$ is the polygamma function, defined in Abramowitz and Stegun ([16], p. 258), we simply apply the chain rule:

$$
\frac{\partial}{\partial x} \ln \Gamma(x+k)=-\gamma+\sum_{r=1}^{\infty}\left(\frac{1}{r}-\frac{1}{r+x+k-1}\right)
$$

where $\gamma$ is the Euler-Mascheroni constant.

The ML estimation of $\Theta, \hat{\Theta}$, requires solving the non-linear system $U(\mathbf{x}, \Theta)=0$, which does not lead to a closed-form expression for $\hat{\Theta}$. Then we require numerical methods to estimate $\Theta$. An asymptotic expansion of $\psi_{0}(x)$ is provided in Abramowitz and Stegun ([16], p. 259), and by using the first order approximation $\psi_{0}(x) \simeq \ln x-(1 / 2 x)$ in $\left(17\right.$ and 18), we obtain the ML estimation of $\Theta=(i, j, \theta, \mu, \Omega)^{T}$ as a solution of the following fixed-point type equations;

$$
g(\hat{i})=\hat{i}, g(\hat{j})=\hat{j}, g(\hat{\theta})=\hat{\theta}, g(\hat{\mu})=\hat{\mu} \text {, and } g(\hat{\Omega})=\hat{\Omega} .
$$

This solution can be obtained by simple iterative procedure, for instance suppose we start with an initial guess $\hat{\mu}_{0}$, then the next iteration $\mu_{1}$ can be obtained as $\hat{\mu}_{1}=g\left(\hat{\mu}_{0}\right)$, similarly, $\hat{\mu}_{2}=g\left(\hat{\mu}_{1}\right)$ and so on. Finally the iterative procedure should be stopped when $\left|\hat{\mu}_{i}-\hat{\mu}_{i+1}\right|<\varepsilon$, where $\varepsilon$ is a pre-assigned tolerance value. Also, to compute the standard error, approximate confidence intervals and hypothesis testing of $\Theta$, we use the information matrix that does not have a closed form. Then we use observed information matrix, defined as $\varsigma\left(\Theta^{*}\right)=$ $-\left.\nabla \nabla^{T} l_{n}\right|_{\Theta=\Theta^{*}}$.

Also,

$$
\hat{H}_{X}\left(f_{\mathbf{X}^{w}}(\mathbf{x})\right)=\ln 2+\left(\hat{\mu}+\frac{\hat{\theta}}{2}\right)[\ln (\hat{\mu} / \hat{\Omega})-1]+\frac{(2 \hat{\mu}+\hat{\theta}-1)}{2}\left[\psi\left(\hat{\mu}+\frac{\hat{\theta}}{2}\right)-\ln \left(\frac{\hat{\mu}}{\hat{\Omega}}\right)\right]-\ln \Gamma\left(\hat{\mu}+\frac{\hat{\theta}}{2}\right)
$$

and

$$
\hat{H}_{\beta}\left(f_{\mathbf{X}^{w}}(\mathbf{x})\right)=\frac{1}{\beta-1}\left[1-\frac{B\left(\hat{j} \beta+1, \beta\left(\hat{i}+\frac{\hat{\theta}}{2 \hat{\mu}}\right)\right)}{B^{\beta}\left(\hat{j}+1, \hat{i}+\frac{\hat{\theta}}{2 \hat{\mu}}+1\right)}\right] .
$$

According Migon et al. [17] we can treat MLE as approximately hepta-variate normal; consequently, when $n \rightarrow \infty$ we can compute the standard error and asymptotic confidence intervals for $\Theta$ by using the expected fisher information matrix $\left(E\left(\mathbf{J}_{\Theta}\right)\right)$, given by

$$
E\left(\mathbf{J}_{\Theta}\right)=\left[\begin{array}{ccc}
\hat{J}_{11} & \ldots & \hat{J}_{15} \\
& \ddots & \vdots \\
& & \hat{J}_{55}
\end{array}\right],
$$


where elements of this matrix $\left(J_{\Theta}\right)$ based on a single observation is given in Appendix. Also, the asymptotic joint distribution of the maximum likelihood of $\hat{\Theta}$ can be stated as

$$
\sqrt{n}\left[\begin{array}{c}
i-\hat{i} \\
j-\hat{j} \\
\theta-\hat{\theta} \\
\mu-\hat{\mu} \\
\Omega-\hat{\Omega}
\end{array}\right] \stackrel{d}{\rightarrow} N_{5}\left(0, E\left(\mathbf{J}^{-1}\right)\right)
$$

where $\stackrel{d}{\rightarrow}$ denotes convergence in distribution and $\mathbf{J}^{-1}$ is the inverse of the fisher information matrix $\mathbf{J}$ with

$$
\frac{1}{n} \mathbf{J}^{-1}=\frac{1}{n}\left[\begin{array}{ccc}
\hat{J}_{11} & \ldots & \hat{J}_{15} \\
& \ddots & \vdots \\
& & \hat{J}_{55}
\end{array}\right]^{-1}=\frac{1}{n}\left[\begin{array}{cccc}
\operatorname{Var}(\hat{i}) & \operatorname{Cov}(\hat{i}, \hat{j}) & \cdots & \operatorname{Cov}(\hat{i}, \hat{\theta}) \\
& \operatorname{Var}(\hat{j}) & \ldots & \operatorname{Cov}(\hat{j}, \hat{\theta}) \\
& \ddots & \vdots \\
& & & \operatorname{Var}(\hat{\theta})
\end{array}\right] .
$$

Then asymptotic equal tailed $100(1-\kappa) \%$ confidence intervals for $\Theta$ can be determined as:

$$
\hat{\imath} \pm z_{\kappa / 2} \sqrt{\operatorname{Var}(\hat{\imath})} ; \hat{\jmath} \pm z_{\kappa / 2} \sqrt{\operatorname{Var}(\hat{\jmath})} ; \hat{\mu} \pm z_{\kappa / 2} \sqrt{\operatorname{Var}(\hat{\mu})} ; \hat{\Omega} \pm z_{\kappa / 2} \sqrt{\operatorname{Var}(\hat{\Omega})} ; \hat{\theta} \pm z_{\kappa / 2} \sqrt{\operatorname{Var}(\hat{\theta})}
$$

where $z_{\mathcal{K}}$ is $100 \kappa$ th percentile of $N(0,1)$.

\subsection{Simulation}

To generate NA distributed samples, we can use the relationship between NA random variable $(X)$, and gamma random variable $(Y)$, that is $Y=X^{2}$, for generating gamma distributed samples, we used Matlab programming. Without loss of generality throughout the simulations, we generated data with $i=j=0$, and $\theta=2$ for $\Phi=\left(\mu, \Omega, H, H_{\beta}\right)$ that appear in (16), (19) and (20) by using the Monte Carlo simulation as follows.

1. Generate random samples of sizes $\mathrm{n}=25,50,75,100$ for each choice of the vector of the parameters $\Theta=\left(\mu, \Omega, H, H_{\beta}\right)$

2. The estimates are obtained by maximizing (16) numerically.

3. The bias and mean square errors (MSE) of the estimations are calculated based on 1000 Monte Carlo repetitions. and the results are presented in Tables 2 and 3.

4. From Tables 2 and 3, we see that in most of the considered cases, the MSE of the estimation parameters decrease as $n$ increases. The first 1000 simulations of the estimates and their biases are plotted in Figures 3 and 4.

The box plot in Figure 3 shows that among 1000 simulated estimates, there are 11 outliers for estimating $\mu$, six outliers for estimating $\Omega$, five outliers for estimating $H$ and ten outliers for estimating $H_{\beta}$. The probability plots in Figure 4 show that the biases of estimates, follow normal distributions that shown the data fit the GN model well, except the outliers are evident at the high and low end of the range.

Table 2 Bias of the estimation of $\hat{\Phi}=\left(\hat{\mu}, \hat{\Omega}, \hat{H}, \hat{H}_{\beta}\right)$.

\begin{tabular}{lccccc}
\hline $\boldsymbol{\Phi}=\left(\boldsymbol{\mu}, \boldsymbol{\Omega}, \boldsymbol{H}, \boldsymbol{H}_{\boldsymbol{\beta}}\right)$ & $\boldsymbol{n}$ & $\hat{\boldsymbol{\mu}}$ & $\hat{\boldsymbol{\Omega}}$ & $\hat{\boldsymbol{H}}$ & $\hat{\boldsymbol{H}}_{\boldsymbol{\beta}}$ \\
\hline & 25 & 1.66471 & -0.0097242 & 0.1288861 & 0.3051938 \\
$(9,0.05,1.9,0.9)$ & 50 & 0.83921 & -0.0097206 & 0.1069311 & 0.2479989 \\
& 75 & 0.78625 & -0.0092648 & 0.1165099 & 0.0756641 \\
& 100 & 0.49690 & -0.0097457 & 0.0977865 & 0.1894217 \\
& 25 & 6.35943 & 0.25078738 & -0.6844654 & 0.1247769 \\
$(21,0.3,1.4,0.997)$ & 50 & 5.34456 & 0.25220518 & -0.699643 & 0.0948707 \\
& 75 & 4.06696 & 0.23026453 & -0.8001621 & 0.4420531 \\
& 100 & 4.90382 & 0.25265919 & -0.7125854 & 0.0991315 \\
\hline
\end{tabular}


Table $3 \mid$ MSE of $\hat{\Phi}=\left(\hat{\mu}, \hat{\Omega}, \hat{H}, \hat{H}_{\beta}\right)$.

\begin{tabular}{lccccc}
\hline $\boldsymbol{\Phi}=\left(\boldsymbol{\mu}, \boldsymbol{\Omega}, \boldsymbol{H}, \boldsymbol{H}_{\boldsymbol{\beta}}\right)$ & $\boldsymbol{n}$ & $\hat{\boldsymbol{\mu}}$ & $\hat{\boldsymbol{\Omega}}$ & $\hat{\boldsymbol{H}}$ & $\hat{\boldsymbol{H}}_{\boldsymbol{\beta}}$ \\
\hline & 25 & 17.39353 & 0.000134 & 0.057585 & 2.794999 \\
$(9,0.05,1.9,0.9)$ & 50 & 6.51417 & 0.000121 & 0.035095 & 2.153815 \\
& 75 & 3.470943 & $8.87 \mathrm{E}-05$ & 0.019668 & 0.005767 \\
& 100 & 3.316798 & 0.000114 & 0.026113 & 1.46267 \\
& 25 & 66.02313 & 0.063837 & 0.499707 & 0.141606 \\
$(21,0.3,1.4,0.997)$ & 50 & 37.24665 & 0.063766 & 0.498175 & 0.009003 \\
& 75 & 36.05122 & 0.059743 & 0.752393 & 1.621199 \\
& 100 & 28.44723 & 0.064003 & 0.514119 & 0.028294 \\
\hline
\end{tabular}

MSE: mean square errors.

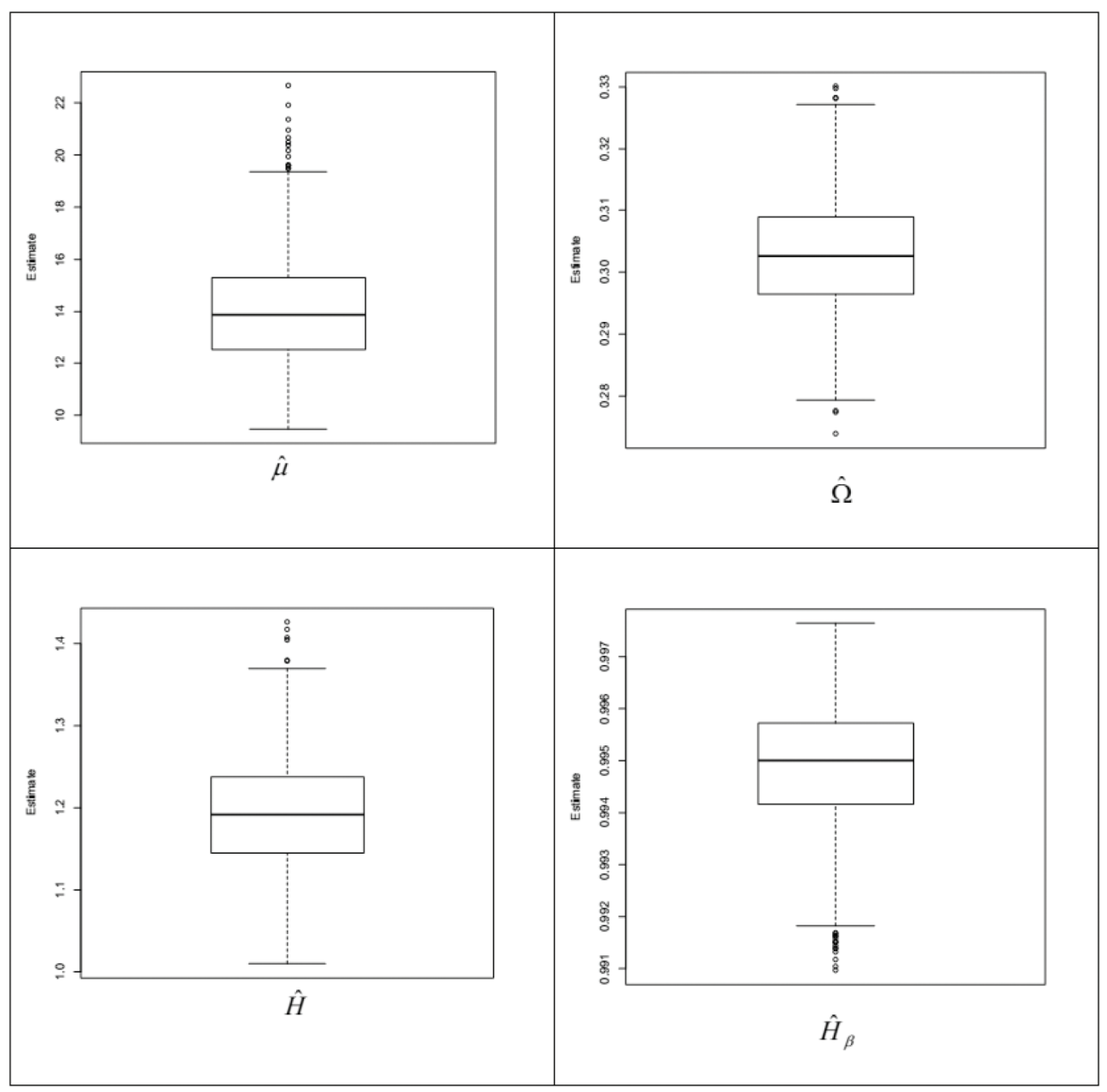

Figure 3 Box plot of the estimates $\hat{\Phi}=\left(\hat{\mu}, \hat{\Omega}, \hat{H}, \hat{H}_{\beta}\right)$.

\section{APPLICATIONS}

\subsection{Applications in Reliability}

In next results, The application of the proposed model will be verified in the reliability theory based on three datasets. The GN distribution is compared with other usual two parameter lifetime distributions. The following lifetime distributions were considered.

\subsection{Wave Height Data}

The modeling of wave heights is used by mariners, as well as in coastal, ocean and naval engineering. Prevosto et al. [18], Mathiesen et al. [19] and Goda and Kobune [20] studied the heights of waves by RA distribution. In this subsection, an application of the NA distribution to 
this problem is described. The set of data we use are for the maximum down-crossing wave heights (Hmax D), in meters, for 23 abnormal waves, as reported by Petrova et al. ([21], p. 237). These waves were measured at the offshore platform North Alwyn in the northern part of the North Sea, about 100 miles east the Shetland Islands, during November storm in 1997.

The data are presented below:

Data set: $16.44,18.17,16.97,13.51,15.19,17.63,17.99,20.29,18.09,11.7,10.78,13.17,13.32,19.92,18.33,19.44,15.62,16.43,16.01,13.46$, $15.0,13.47,14.09$.

In order to compare the four distribution models, we consider the criteria like AIC (Akaike information criterion), AICC (corrected Akaike information criterion) and BIC (Bayesian information criterion). The better distribution corresponds to lesser AIC, AICc and BIC values. Table 4 indicated that the GN distribution has the lesser AIC, AICC and BIC values compared to NA distribution, OG distribution and RA distribution. Hence, we can conclude that the GN distribution leads to a better fit than other models. Further, Table 5 reports the maximum likelihood estimates of the parameters, also, 95\% asymptotic confidence intervals for the parameters are provided. Moreover, Figure 5 shows empirical and fitted distribution, we see that the GN provided a better fit for this data that NA distribution, OG distribution and RA distribution.
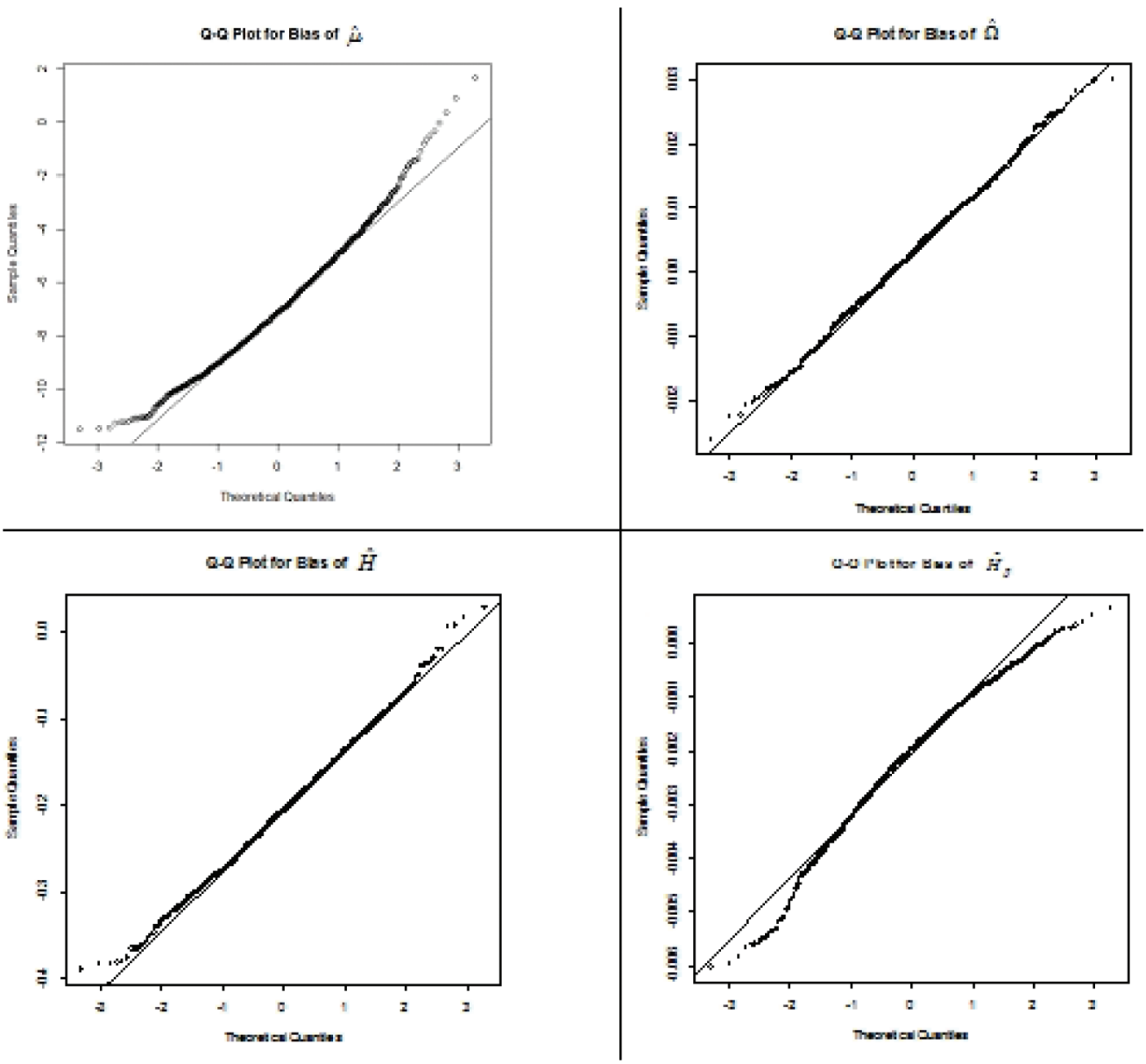

Figure $4 \mid Q-Q$ plot for the bias of $\hat{\Phi}=\left(\hat{\mu}, \hat{\Omega}, \hat{H}, \hat{H}_{\beta}\right)$.

Table 4 Goodness of fit criteria.

\begin{tabular}{lllll}
\hline Model & $\mathbf{- 2} \log \boldsymbol{L}$ & AIC & AICc & BIC \\
\hline GN & 100.277 & 104.277 & 104.877 & 106.548 \\
NA & 140.833 & 144.833 & 145.433 & 147.104 \\
OG & 202.2293 & 204.2293 & 204.4198 & 205.3648 \\
RA & 217.1838 & 219.1838 & 219.3743 & 220.3193 \\
\hline
\end{tabular}

AIC: Akaike information criterion; AICC: corrected Akaike information criterion; BIC: Bayesian information criterion; GN: Generalized Nakagami; NA: Nakagami; OG: One-sided Gaussian distribution; RA: Rayleigh distribution. 
Table 5 Estimate of MLEs for wave data under four particular sub-models $G N(0,0,2, \mu, \Omega)$ and asymptotic confidence intervals are in parentheses.

\begin{tabular}{lcccc}
\hline Model & $\hat{\boldsymbol{\mu}}$ & $\hat{\mathbf{\Omega}}$ & $\hat{\boldsymbol{H}}$ & $\hat{\boldsymbol{H}}_{\boldsymbol{\beta}=2}$ \\
\hline \multirow{2}{*}{ GN } & 9.879034 & 234.762957 & -2.301852 & 0.9997 \\
NA & $(-2.34805,22.10612)$ & $(165.8062,303.7197)$ & & 0.9455498 \\
OG & 9.499 & 258.526697 & 2.36844 & 0.9896195 \\
RA & $(-4.4774,23.4757)$ & $(169.7344,347.319)$ & & \\
& 1 & $(-40.927,1517.95165)$ & 4.02811 & 0.9918437 \\
\hline
\end{tabular}

GN: Generalized Nakagami; MLE: maximum likelihood estimator; NA: Nakagami; OG: One-sided Gaussian distribution; RA: Rayleigh distribution.

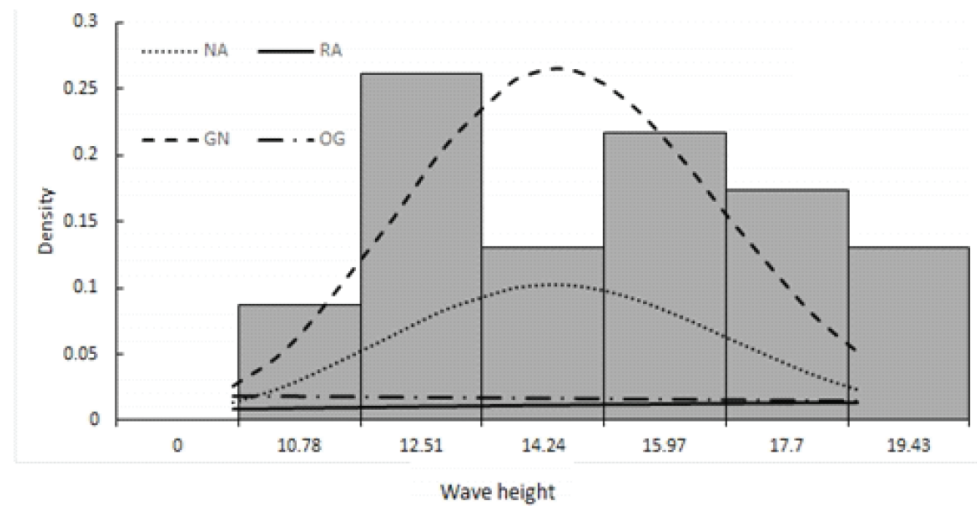

Figure 5 Empirical and fitted distributions.

\section{CONFLICT OF INTEREST}

Authors have no conflict of interest to declare.

\section{REFERENCES}

1. I.E. Telatar, Eur. Trans. Telecommun. 10 (1999), 585-595.

2. J.J. Heckman, Econometrica. 47 (1979), 153-161.

3. J.B. Copas, H.G. Li, J. R. Stat. Soc. B. 59 (1997), 55-95.

4. R.B. Arellano-Valle, M.D. Branco, M.G. Genton, Can. J. Stat. 34 (2006), 581-601.

5. J. Signer, J. Fieberg, T. Avgar, Ecosphere. 8 (2017), e01771.

6. N. Balakrishnan, M.V. Koutras, F.S. Milienos, Commun. Stat. Theory Methods. 47 (2018), 4297-4310.

7. H. Zamani, N. Ismail, M. Shekari, J. Biostat. Epidemiol. 4 (2018), 18-23.

8. M. Arashi, A. Bekker, J. Van Niekerk, Linear Algebra Appl. 561 (2019), 24-40.

9. G.P. Patil, in: A.H. El-Shaarawi, W.W. Piegorsch (Eds.), Encyclopedia of Environmetrics, vol. 4, John Wiley \& Sons, Chichester, England, 2002.

10. A. Azzalini, Scand. J. Stat. 12 (1985), 171-178.

11. I.S. Gradshteyn, I.M. Ryzhik, in: A. Jeffrey, D. Zwillinger (Eds.), Table of Integrals, Series, and Products, sixth ed., Academic Press, New York, NY, USA, 2000.

12. S. Nadarajah, S. Kotz, Math. Probl. Eng. 4 (2004), 323-332.

13. E.D. Rainville, Special Functions, Chelsea Publ. Co., Bronx, NY, USA, 1971.

14. N. Ebrahimi, F. Pellerey, J. Appl. Probab. 32 (1995), 202-202.

15. J. Havrda, F. Charvat, Kybernetika. 3 (1967), 30-35.

16. M. Abramowitz, I.A. Stegun, Handbook of Mathematical Functions with Formulas, Graphs, and Mathematical Tables, Dover, New York, NY, USA, 1964.

17. H.S. Migon, D. Gamerman, F. Louzada, Statistical Inference: an Integrated Approach, London, England, 2014.

18. M. Prevosto, H.E. Krogstad, A. Robin, Coast. Eng. 40 (2000), 329-360.

19. M. Mathiesen, Y. Goda, P.J. Hawkes, E. Mansard, M.J. Martin, E. Peltier, E.F. Thompson, G. Van-Vledder, J. Hydraul. Res. 32 (1994), $803-814$.

20. Y. Goda, K. Kobune, in Proceedings of the International Conference on Coastal Engineering, Delft, The Netherlands, 1990.

21. P. Petrova, Z. Cherneva, C.G. Soares, Appl. Ocean Res. 28 (2006), 235-245. 


\section{APPENDIX}

1. $\bar{\varpi}_{\mu, j, i}(n, k)$ that appear in (13):

$$
\varpi_{\mu, j, i}(n, k)=a_{\mu, j, i}(n, k) b_{\mu, j, i}(n, k)
$$

where

$$
\begin{gathered}
a_{\mu, j, i}(n, k)=(-1)^{k-n^{2}}\left(\begin{array}{l}
j \\
k
\end{array}\right)(\Gamma(\mu))^{-(k+i+1)}(\mu)^{-(k+i)}((k+i-1) !)^{-1} ; \\
b_{\mu, j, i}(n, k)=(n+1)_{(k+i-1)}(\mu / \Omega)^{\mu(k+i)+\mu+n^{2}}\left((\mu)_{n} / n !(\mu+1)_{n}\right)^{n} ; \\
\bar{\varpi}_{\mu, j, i}(n, k)=\varpi_{\mu, j, i}(n, k)\left(\frac{(\mu)_{n}}{n !(\mu+1)_{n}}\right)^{n} /\left([(\mu / \Omega)]^{\mu(k+i)+\mu+\frac{\theta}{2}+n^{2}} I_{i, j, \theta}(\mu, \Omega)\right) .
\end{gathered}
$$

2. The fixed-point type equations for $\Theta=(i, j, \mu, \Omega, \theta)^{T}$ :

$$
\begin{aligned}
& g(\hat{i})=\exp \left\{\alpha_{1}+\ln \alpha_{2}+\frac{j+1}{2 \alpha_{3} \alpha_{2}}\right\}-\frac{\theta}{2 \mu}-1, \\
& g(\hat{j})=1 /\left[c_{1}+2 \ln \Gamma(\mu)-2 \psi_{0}\left(\pi_{4}+1\right)+2 \ln (j+1)\right]-1, \\
& g(\hat{\theta})=1 /\left(\begin{array}{c}
\left(\left[\sum_{k=1}^{N} \ln x_{k}-(N \ln \Omega / 2)-N \ln \mu(1-\mu) / 2 \mu-N \ln \Gamma(\mu) / 2 \mu\right] / N\right) \\
-2 \mu\left(-\gamma+\sum_{r=1}^{\infty}\left(\frac{1}{r}-\frac{1}{r+i+\frac{\theta}{2 \mu}-1}\right)\right) \\
+\left[2 \mu\left(j+i+\frac{\theta}{2 \mu}+1\right)\right]^{-1}+\left(2 \mu\left(j+i+\frac{\theta}{2 \mu}\right)\right)^{-1} \\
+2 \mu\left(-\gamma+\sum_{r=1}^{\infty}\left(\frac{1}{r}-\frac{1}{r+j+i+\frac{\theta}{2 \mu}-1}\right)\right)
\end{array}\right)-2 \mu i, \\
& g(\hat{\mu})=\left(\left\{\begin{array}{c}
i \pi_{1}+N j \pi_{2}+2 \pi_{3}-\pi_{6}-N \ln \Omega+\left(\theta N / 2 \mu^{2}\right) \ln \Gamma(\mu)-N \psi(\mu)\left(\pi_{4}\right) \\
+N\left(\theta / 2 \mu^{2}\right)\left[\psi_{0}\left(\pi_{4}+j\right)-\psi_{0}\left(\pi_{4}+1\right)\right]
\end{array}\right\} / 2 N \pi_{5}\right)^{1 / 2},
\end{aligned}
$$

and

$$
g(\hat{\Omega})=N\left(\frac{\theta}{2}+\mu\right)\left((\mu / \Omega) \pi_{6}-i \Omega^{2 \mu-2} \mu^{-\mu+2} \sum_{k=1}^{N} \frac{x_{k}^{2(1-\mu)} C_{k}}{\gamma\left(\mu, \mu x_{k}^{2} / \Omega\right)}+j N \Omega^{2 \mu-2} \mu^{-\mu+2} \frac{\sum_{k=1}^{N} C_{k} x_{k}^{2(1-\mu)}}{\zeta}\right),
$$

where

$$
\begin{aligned}
& \alpha_{1}=\frac{1}{N} \sum_{k=1}^{N} \ln \gamma\left(\mu, \mu x_{k}^{2} / \Omega\right)-\ln \Gamma(\mu), \\
& \alpha_{2}=i+\frac{\theta}{2 \mu}+j+2, \\
& \alpha_{3}=i+\frac{\theta}{2 \mu}+1, \\
& c_{1}=-2 \sum_{k=1}^{N} \ln \zeta / N, \\
& \pi_{1}=\sum_{k=1}^{N}(\mu)^{\mu-1}\left(x_{k}^{2} / \Omega\right)^{\mu} \exp \left(-\mu x_{k}^{2} / \Omega\right) / \gamma\left(\mu, \mu x_{k}^{2} / \Omega\right), \\
& \pi_{2}=\left[\psi(\mu) \Gamma(\mu)-(\mu)^{\mu-1} \sum_{k=1}^{N}\left(x_{k}^{2} / \Omega\right)^{\mu} \exp \left(-\mu x_{k}^{2} / \Omega\right)\right] / \zeta, \\
& \pi_{3}=\sum_{k=1}^{N} \ln x_{k}, \\
& \pi_{4}=i+j+\frac{\theta}{2 \mu}+1, \\
& \pi_{5}=\left[\left\{\ln \mu\left(-4 \mu^{2}-2 \theta\right) / 2\right\}+\theta(1-\mu)-2 \mu^{2}\right] \text { and } \\
& \pi_{6}=\sum_{k=1}^{N} x_{k}^{2} / \Omega .
\end{aligned}
$$


3. The elements of the $5 \times 5$ unit expected information matrix are given by

$$
\begin{aligned}
& J_{11}=N\left\{\psi_{i}^{t r}\left(\pi_{4}+1\right)-\psi_{i}^{t r}\left(\alpha_{3}\right)\right\}, J_{12}=J_{21}=N \psi_{j}^{t r}\left(\pi_{4}+1\right), \\
& J_{13}=J_{31}=-\sum_{k=1}^{N} \varpi C_{k}\left(x_{k}^{2} / \Omega\right)^{-\mu+1}-N\left[\psi_{\mu}^{t r}\left(\alpha_{3}\right)-\psi_{\mu}^{t r}\left(\pi_{4}+1\right)+\psi_{0}(\mu)\right], \\
& J_{14}=J_{41}=-\sum_{k=1}^{N} \varpi(1 / \Omega)^{-2 \mu+3} C_{k} \mu^{-\mu+2}\left(x_{k}^{2}\right)^{-\mu+1}, \\
& J_{15}=J_{51}=N\left(2 \mu^{-1}\left(i+\frac{\theta}{2 \mu}\right)^{-2}+2 \mu \sum_{r=1}^{\infty}\left(r+i+\frac{\theta}{2 \mu}-1\right)^{-2}+b_{1}\right) \text {, } \\
& J_{22}=N\left\{\psi_{j}^{t r}\left(\pi_{4}+1\right)-\psi_{j}^{t r}(j+1)\right\}, J_{23}=J_{32}=b_{2}-N \psi_{0}(\mu)+N \psi_{\mu}^{\text {tr }}\left(\pi_{4}+1\right), \\
& J_{24}=J_{42}=\zeta^{-1} N \Omega^{2 \mu-3}(\mu)^{-\mu+2} \sum_{k=1}^{N} C_{k} x_{k}^{-2(\mu+1)}, \\
& J_{25}=J_{52}=2 \mu N \sum_{r=1}^{\infty}\left(r+\pi_{4}-2\right)^{-2}-2 \mu N\left[2 \mu\left(\pi_{4}\right)\right]^{-2}-2 \mu N\left(2 \mu\left(\pi_{4}-1\right)\right)^{-2}, \\
& i \sum_{k=1}^{N} \varpi^{2} C_{k}\left[\varpi^{-1} b_{3}-\mu^{\mu-1}\left(x_{k}^{2} / \Omega\right)\right]+N j \zeta^{-3}\left(b_{4}-\sum_{k=1}^{N} C_{k} b_{3}-\zeta b_{5}\right) \\
& -N b_{6}+\psi_{0}(\mu) \frac{\theta N}{2 \mu^{2}}-\ln \Gamma(\mu) \frac{\theta N}{\mu^{3}}-N b_{7}+N b_{8}, \\
& J_{34}=J_{43}=i \sum_{k=1}^{N} \varpi^{2}\left(\varpi^{-1} C_{k}\left(\mu / \Omega^{2}\right)\left(x_{k}^{2}-\Omega\right)+\mu^{-\mu+2} \Omega^{2 \mu-3} C_{k}^{2} x_{k}^{-2(\mu+1)}\right) \\
& +N j \zeta^{-2}\left(-\zeta\left(\mu / \Omega^{2}\right) \sum_{k=1}^{N} C_{k} x_{k}\left(x_{k}^{2}-\Omega\right)+b_{9}\right)+\sum_{k=1}^{N} x_{k}^{2} / \Omega^{2}-N \psi_{0}(\Omega), \\
& J_{35}=J_{53}=N \mu^{-2} b_{10}-N\left[b_{11}+b_{12}+b_{13}\right], \\
& J_{44}=\sum_{k=1}^{N}-2 \mu x_{k}^{2} / \Omega^{3}+\frac{N}{\Omega^{2}}\left(\frac{\theta}{2}+\mu\right) \\
& -i \sum_{k=1}^{N} \varpi^{2}(\Omega)^{2 \mu-3}(\mu)^{-\mu+2} C_{k}\left(x_{k}^{2}\right)^{1-\mu}\left[(\mu-2)+\left(\mu x_{k}^{2}\right)(\Omega)\right] \\
& -i \sum_{k=1}^{N} \varpi^{2}(\Omega)^{4 \mu-5}(\mu)^{2(2-\mu)} C_{k}^{2}\left(x_{k}^{2}\right)^{-2 \mu+2} \\
& +\frac{j N}{\zeta 2}\left[\zeta \mu^{-\mu+2} \Omega^{2 \mu-3} \sum_{k=1}^{N} C_{k} x_{k}^{2(1-\mu)}\left[(\mu-2)+(1 / \Omega)\left(\mu x_{k}^{2}\right)\right]\right] \\
& -\frac{j N \Omega^{4 \mu-5} \mu^{-2 \mu+4}}{\zeta^{2}} \sum_{k=1}^{N} C_{k} x_{k}^{-2 \mu+1} \sum_{k=1}^{N} C_{k} x_{k}^{2(1-\mu)}, \\
& J_{45}=-\frac{N}{2 \Omega} \text {, and } J_{55}=-N\left[\begin{array}{c}
-\left(2 \mu\left(\alpha_{3}-1\right)\right)^{-2}+\sum_{r=1}^{\infty}\left(r+\alpha_{3}-2\right)^{-2}+\left[2 \mu\left(\pi_{4}\right)\right]^{-2} \\
+\left(2 \mu\left(\pi_{4}-1\right)\right)^{-2}-\sum_{r=1}^{\infty}\left(r+\pi_{4}-2\right)^{-2}
\end{array}\right] \text {, }
\end{aligned}
$$

where $\varpi^{i}=1 / \gamma^{i}\left(\mu, \mu x_{k}^{2} / \Omega\right), b_{1}=2 \mu \sum_{r=1}^{\infty}\left(r+\pi_{4}\right)^{-2}-2 \mu\left(2 \mu \pi_{4}\right)^{-2}-2 \mu\left(2 \mu\left(\pi_{4}-1\right)\right)^{-2}, \quad b_{2}=\sum_{k=1}^{N} \zeta^{-1}$ $\left(\Gamma(\mu) \psi_{0}(\mu)-\sum_{k=1}^{N} C_{k}\left(x_{k}^{2} / \Omega\right)^{1-\mu}\right), b_{3}=\ln \mu+\ln \left(x_{k}^{2} / \Omega\right)-\left(x_{k}^{2} / \Omega\right), b_{4}=\psi_{\mu}^{\text {tr }}(\mu) \Gamma(\mu)+\left(\psi_{0}(\mu)\right)^{2}, b_{5}=\left[\psi(\mu) \Gamma(\mu)-\sum_{k=1}^{N} C_{k}\right] \times$ 
$\left[\psi_{0}(\mu)-\sum_{k=1}^{N} C_{k}\left(x_{k}^{2} / \Omega\right)^{-\mu+1}\right], b_{6}=2 \mu^{-1} \ln \mu\left(4 \mu^{2}+2 \theta\right)+2^{-1} \mu^{-3} \theta(\mu-2)-8 \mu \ln \mu-4 \mu^{2} \psi_{0}(\mu)-2 \theta \psi_{0}(\mu)$,

$b_{7}=(\theta / 2) \mu^{-1} \psi_{\mu}^{\text {tr }}(\mu)-(\theta / 2) \mu^{-2} \psi_{0}(\mu), b_{8}=-\theta \mu^{-3} \psi_{0}\left(\alpha_{3}\right)+(\theta / 2) \mu^{-2} \psi_{\mu}^{\text {tr }}\left(\alpha_{3}\right)+\theta \mu^{-3} \psi_{0}\left(\pi_{4}+1\right)+(\theta / 2)^{2} \mu^{-4} \psi_{\mu}^{\text {tr }}\left(\pi_{4}+1\right)$, $b_{9}=\left(\psi(\mu) \Gamma(\mu)-\sum_{k=1}^{N} C_{k}\right)\left(\mu^{2-\mu} \sum_{k=1}^{N} C_{k}\left(x_{k}^{2} / \Omega^{2}\right)^{1-\mu} \gamma\left(\mu, \mu x_{k}^{2} / \Omega\right)\right)$,

$b_{10}=\left(\mu+\ln \mu-\mu \Psi_{0}(\mu)+\ln \Gamma(\mu)-1\right) / 2, b_{11}=-2 \gamma+2 \sum_{r=1}^{\infty} r^{-1}-\left(r+i+(\theta / 2) \mu^{-1}-1\right)^{-1}-\left(1 / 2 \mu^{2}\left(\alpha_{3}-1\right)\right)$,

$b_{12}=\left(1 / 2 \mu^{2} \pi_{4}\right)+2\left(\pi_{4}-1\right)\left(2 \mu\left(\pi_{4}-1\right)\right)^{-2}-(\theta / \mu) \sum_{r=1}^{\infty}\left(\left(r+\alpha_{3}-2\right)^{-2}\right), b_{13}=\frac{\theta}{\mu}\left(\sum_{r=1}^{\infty}\left(r+\pi_{4}-2\right)^{-2}\right)-$ $2\left(-\gamma+\sum_{r=1}^{\infty}\left(\frac{1}{r}-\frac{1}{r+\pi_{4}-2}\right)\right)$. 